\title{
The Role of Portable Incisional Negative Pressure Wound Therapy (piNPWT) in Reducing Local Complications of Post-bariatric Brachioplasty: A Case-Control Study
}

\author{
Federico Facchin ${ }^{1}(\mathbb{D})$ Andrea Pagani $^{2} \cdot$ Paolo Marchica $^{1} \cdot$ Laura Pandis $^{1}$. \\ Carlotta Scarpa $^{1} \cdot$ Tito Brambullo $^{1} \cdot$ Franco Bassetto $^{1} \cdot$ Vincenzo Vindigni $^{1}$
}

Received: 23 September 2020/Accepted: 28 December 2020/Published online: 22 January 2021

(C) The Author(s) 2021

\begin{abstract}
Background Due to the great impact of bariatric surgery on the overweight epidemic, the number of post-bariatric body-contouring procedures is constantly increasing worldwide. The portable incisional negative pressure wound therapy (piNPWT) is a promising medical device for accelerating wounds closure and controlling post-operative complication, which have been shown promising results in post-bariatric population. We aimed to evaluate the role of piNPWT in optimizing wound healing and controlling post-operative complications after a post-bariatric brachioplasty.
\end{abstract}

Federico Facchin

federicofacchin@yahoo.it

Andrea Pagani

andrea.pagani@studenti.unipd.it

Paolo Marchica

paolomarchica91@gmail.com

Laura Pandis

laura.pandis@aopd.veneto.it

Carlotta Scarpa

carlotta.scarpa@unipd.it

Tito Brambullo

tito.brambullo@aopd.veneto.it

Franco Bassetto

franco.bassetto@unipd.it

Vincenzo Vindigni

vincenzo.vindigni@unipd.it

1 Plastic and Reconstructive Surgery Unit, University of Padova, Via Nicolò Giustininani 2, 35128 Padua, Italy

2 Clinic and Policlinic of Plastic and Hand Surgery, Technical University of Munich, Ismaninger Str. 22, 81675 Munich, Germany
Patients and Methods 26 post-bariatric female patients who underwent a brachioplasty followed by either a piNPWT (14 cases) or a standard wound treatment (12 controls) were analyzed. The number of post-operative dressing changes, the rate of local post-operative complications (re-operation, hematoma and serosa development, dehiscence and necrosis), the time to dry as well as the scar quality and hospitalization length were evaluated.

Results None of the patients prematurely stopped treatment with piNPWT due to intolerance. The piNPWT patient group showed a significant lower healing time as well as a significant reduction of the number of post-operative dressing changes and hospital stay. Despite the scarring process was excellent from the functional point of view in the long term, we noticed a higher rate of hyperchromic scarring at 90 days after surgery.

Conclusion The piNPWT is a cost-effective and userfriendly medical tool that increase and promote wound healing. We suggest the use of this device in post-bariatric patients who undergo a brachioplasty, especially if there is the need to minimize the number of post-operative dressing changes.

Level of Evidence IV This journal requires that authors assign a level of evidence to each article. For a full description of these evidence-based Medicine ratings, please refer to Table of Contents or the online Instructions to Authors www.springer.com/00266.

Keywords Body-contouring - Post-bariatric patients . Surgical site complications - Obesity · Brachioplasty . Wound care 


\section{Background}

The epidemic of overweight and obesity represents a significant threat for patients' health and a major challenge for the healthcare system [1]. Bariatric surgery and diet-related weight loss reduce significantly comorbidity of obese patients improving their life expectancy $[2,3]$. However, the accumulation of excess cutaneous tissue severely impacts massive weight loss (MWL) patients' quality of life, preventing them from performing social and physical activity. In addition, patients frequently develop skin fold cutaneous infection $[4,5]$. More of $30 \%$ of the American population is obese and almost 300,000 patients per year undergo bariatric surgery $[6,7]$.

Drastical changes in the BMI modify the structure of soft tissues, leading progressively to excess skin in different body districts [4, 8-10]. The combination of adiposity and/or poor skin tone with increasing skin ptosis guides surgeons in choosing the best technique according to the Pittsburgh Rating Scale. Patients with loose and hanging skin should undergo a brachioplasty procedure associated with liposuction in the presence of severe adiposity $[8,11,12]$. Some authors suggest that a chest extension of skin resection is often required to obtain a smooth axillary profile [13]. In 2017, 18033 arm lift procedures have been performed in the U.S., with a $5235 \%$ increase from 2000 [14].

Among different body areas, the postero-medial region of the arms represents a particular region where adipocutaneous excess of MWL patient accumulate in the distal half due to the progressive loosening of the support of superficial, longitudinal and axillary fascial systems of the proximal half [15].

In addition, surgical scars of brachioplasty are the most commonly exposed by every day clothing if compared to other procedures, representing the heavier surgical burden.

Despite surgery is constantly improving, minor and major complications including seroma, hematoma, infection, lymphocele, numbness, peripheral nerve pain and wound dehiscence are still frequent. Post-operative complications rates are as high as $40 \%$ in many studies, especially in associated multiple procedures (e.g., abdominoplasty, mastopexy) [6, 7].

Given the steep learning curve and the high risks, bodycontouring procedures should be performed in dedicated centers with managed through defined protocols from experienced surgeons.

From the surgical point of view, the deep investing fascia, which envelopes arm musculature, should not be violated during surgery, in order to avoid medial antebrachial cutaneous nerve injuries. An axillary Z-plasty or its sinusoidal variation, can be performed to the proposed skin excision in order to prevent severe contractures and to restore the axillary dome's appearance. Tension free closure should be obtained. Moreover, intraoperative infiltration of diluted epinephrine and liquid warming have been shown to reduce seroma rate formation and intraoperative bleeding [16].

The preoperative management is of paramount importance in preventing complications with metabolic and nutritional homeostasis achieved before surgery. A stable weight and nutritional evaluation should be granted [12]. Finally, a dedicated post-operative management of wide surgical wounds is of key importance to improve the final result.

At present, the piNPWT has been shown effective in improving wound healing in several surgical fields, reducing at the same time risks of complications even in other surgeries: inguinal dissection [17], mastectomy [18], ALT flap donor site [19], cesarean sections in highrisk patients $[20,21]$. Benefits from the piNPWT application have been reported in a case-control study in postbariatric abdominoplasty [21] and in cost-utility analysis [22].

Altogether, by assuming that this dressing could act as effective, user-friendly and cost-effective, we compared the effect of piNPWT with a traditional dressing change in MWL brachioplasty patients.

\section{Patients and Methods}

The study was conducted in accordance with the World Medical Association Declaration of Helsinki (June 1964) and subsequent amendments.

We performed a case-control study with 26 post-bariatric female patients (middle age 45.33) who underwent a brachioplasty at the Division of Plastic Surgery, at our University-Hospital, from June 2018 to March 2020. Fourteen patients (middle age 49.33) were treated post operatively with bilateral piNPWT (piNPWT patients) Fig. 1 (PICO, Smith and Nephew, Watford, UK) with a negative aspiration pressure of $-80 \mathrm{mmHg}$. Twelve patients (middle age 41.66) were treated with a traditional dry dressing (TDD group).

Inclusion criteria:

- Massive weight loss at least of 7 points BMI (post MWL surgery or diet reduction)

- Stable weight and metabolic/nutritional homeostasis for at least 6 months [12]

- Female 


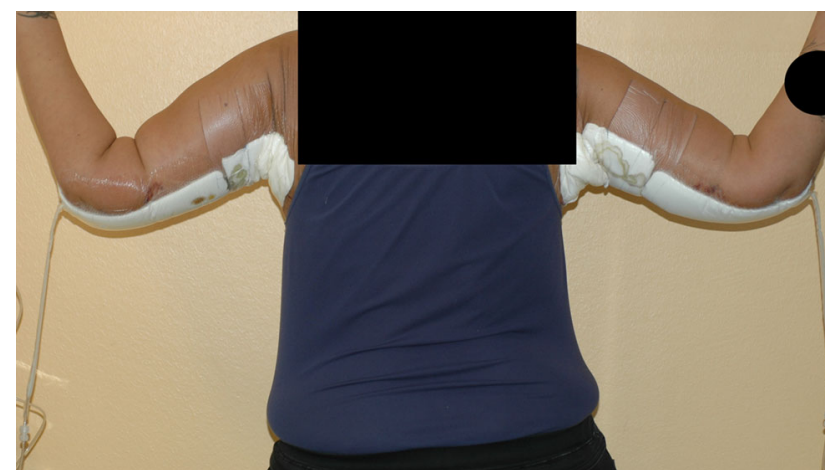

Fig. 1 Post-operative dressing with bilateral piNPWT (piNPWT patients)

Exclusion criteria:

- Unstable weight loss

- Previous arm surgery

- Allergy to glue and tape

- Neurologic, psychiatric or vascular disorders of the upper extremity

- Lymphedema of the arms

- Unrealistic patient expectations

- Raynaud's disease, connective tissue disorders and advanced rheumatoid arthritis [13, 23-25]

Before surgery, a physical examination was performed, evaluating upper limb function, including ROM at shoulder/elbow/hand and grip strength. Authors assessed for excess fat and skin, overall skin quality and tone.

A watertight layered and subcuticular tension free suture was obtained, and a drain per arm was placed in all patients. Both groups used compressive sleeve garments for 30 days after treatment [16]. Dressings were evaluated daily during the hospital stay. Whereas piNPWT was changed after 7 days post operatively, traditional dressings were changed every 3 days after treatment according to our protocol. Patients were instructed to return for follow-up at 90 days.

During every single dressing, the presence of post-operative complications (i.e., blistering, hematoma, serosa, hypertrophic or hyperchromic scars), the number of postoperative dressing change, the hospitalization length and the eventual impact of the associated surgeries were evaluated. Complications were calculated per arm, defined as either hematoma, wound dehiscence, skin necrosis or infection, and classified through Clavien-Dindo classification [26].

The aspect of the scar aspect was evaluated at a mean time of 6 months after surgery with Vancouver Scar Scale [27] (Figs 2, 3).

All procedures have been done in agreement with the declaration of Helsinki.

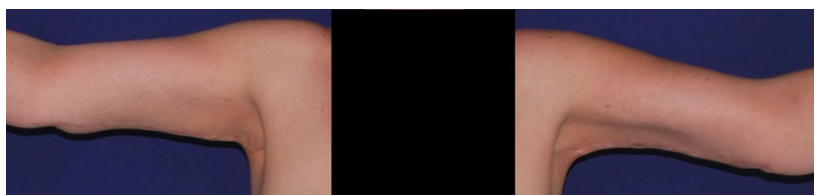

Fig. 2 Post-operative long-term (10 months) follow up of PTT group patient

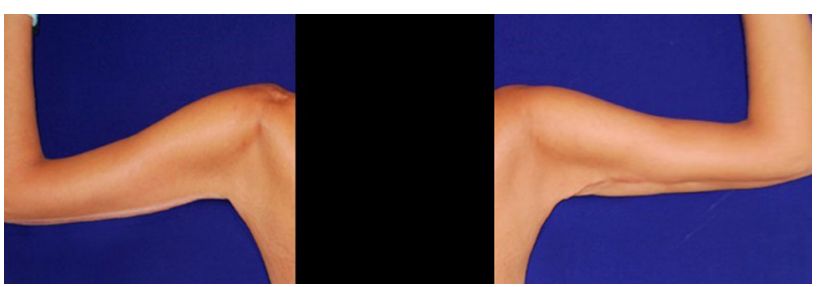

Fig. 3 Post-operative long-term (11 months) follow up of piNPWT group patient

\section{Statistical Analysis}

Sample size was calculated using Pearson's chi-square test to detect meaningful differences (alpha $0.05 ; 1-\beta$ : $90 \%$; enrollment ratio (1) between the two groups. Anticipated effect for the outcome $(-50 \%)$ was based on previous experience and literature on the use of piNPWT in abdominoplasty. Results are expressed as mean \pm SD. An unpaired Student's t Test (GraphPad Prism 7.0, Inc., La Jolla, CA, USA) was used to determine significant differences between groups ( $p$ value $<0.05)$.

\section{Results}

After successfully performing 26 brachioplasty, $n=14$ patients were randomly treated post operatively with a piNPWT and $n=12$ patients with a traditional dressing. (Fig. 4). Among the investigated patients, we did not observe significant differences in demographics as reported in Table 1 except for BMI loss, which appeared to be higher in the piNPWT group. Data regarding bariatric procedures and previous post-bariatric procedures are reported in Fig. 5. 38,4\% of patients had a previous bodycontouring procedure. Two patients have lost weight without surgery, one of them was excluded from the study because affected by anorexia. Post-operative results and analyzed outcomes are collected in Table 2. As reported in Figs 4,6 , patients of the piNPWT group were treated with brachioplasty and liposuction, 8 patients with the combination of brachioplasty and mastopexy with implant (Polytech Health \& Aesthetics Altheimer Str. 32, 64807 Dieburg, Germany) with three of them managed with arm liposuction as well. Figure 4. No intra-operative 


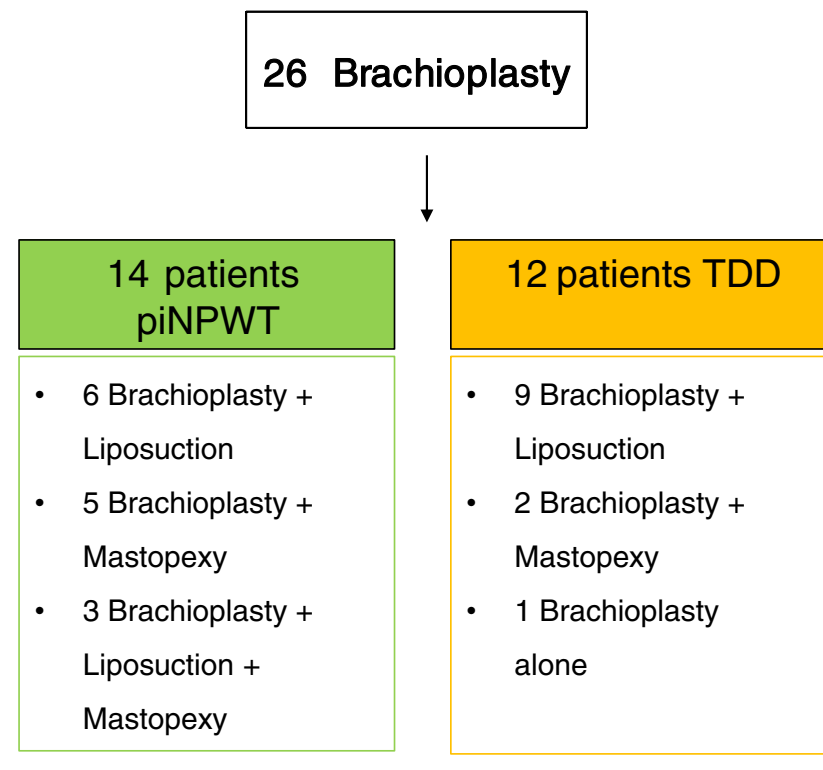

Fig. 4 Study design and groups composition

Table 1 Patients demographics of the two groups

\begin{tabular}{lll}
\hline & piNPWT & TDD \\
\hline Sex & All females & \\
Age (years) * & $43 \pm 11.09$ & $49 \pm 9.32$ \\
Smokers & 3 & 4 \\
Number of obese patient per & 3 & 5 \\
$\quad$ group BMI $>30$ & & \\
Time from bariatric & $3.25 \pm 2.51$ & $5 \pm 4.22$ \\
$\quad$ procedure (years) * & & \\
Weight loss (kg)* & $54.85 \pm 19.73$ & $43.47 \pm 17.80$ \\
BMI before bariatric surg* & $47.52 \pm 7.82$ & $45.04 \pm 4.55$ \\
BMI after bariatric surgery** & $27.03 \pm 2.91$ & $28.92 \pm 4.6$ \\
BMI loss* & $20.49 \pm 6.27$ & $16.12 \pm 6.00$ \\
Ptosis Degree Pittsburg & 5 patients grade 2 & 5 patients grade \\
$\quad$ Rating Scale [8] (El & $(2 \mathrm{~b}) 9$ grade 3 & $2(2 \mathrm{~b}) 7$ grade 3 \\
$\quad$ Khatib [13]) & $(3)$ & $(3)$ \\
Weight of tissue removed & $180.71 \pm 60.94$ & $185.75 \pm 75.98$ \\
per arm (gr)* & & \\
\hline
\end{tabular}

The two groups were comparable when considering the majority of variable analyzed

$* p>0.05$

complications were reported; the piNPWT was generally well tolerated.

As for post-operative complications, one patient of the TDD group developed a wound dehiscence of the right arm suture requiring surgical revision (Clavien-Dindo Grade III) and concomitant blistering of the left arm treated with wet to dry dressing (Clavien-Dindo Grade II). One patient of the piNPWT group developed blistering treated with wet to dry dressing (Clavien-Dindo Grade II). Two patients of the TDD group developed ecchymosis (Clavien-Dindo Grade I). Two hematomas were treated conservatively in the piNPWT and one in the TDD group (Clavien-Dindo Grade I). The patient with hematoma in the TDD group subsequently developed a seroma requiring multiple aspirations (Clavien-Dindo Grade II).

No reported discomfort was associated with the use of piNPWT or dry dressing. Three patients of the piNPWT group reported increased itching that did not require dressing change or suspension of therapy.

The number of post-operative dressing changes was 4.91 \pm 0.79 in the TDD group vs. $2 \pm 0.77$ in the piNPWT group $\left(t\right.$-Test $\left.=4.24 \times 10^{-9} ; p<0.05\right)$. Furthermore, a significant difference was calculated in the "Time to dry" 17.66 \pm 4.79 days in the TDD group vs. $9.36 \pm 2.15$ days in the piNPWT group $\left(t\right.$-Test $\left.=1.52 \times 10^{-5} ; p<0.05\right)$. Finally, a significant difference in the hospitalization length was recorded $5.33 \pm 1.49$ days in the TDD group vs. $3.07 \pm$ 1.14 days in the piNPWT group $(t$-Test $=0.0002 ; p<0.05)$. Seven patients of the piNPWT group developed a hyperchromic scar at 90 days Fig. 6, which then subsides with proper scar management (sun protection and scar massage). The scar clearing was appreciated in subsequent follow-up visits at six, twelve months.

The aspect of the scar was comparable in the two groups with a mean value of the Vancouver Rating Scale at six months of $4.17 \pm 1.99$ and $4.07 \pm 2.49$ for the TDD and piNPWT groups, respectively ( $p$ value $>0.05)($ Figs 2,3$)$.

\section{Discussion}

MWL patients are at higher risk for developing complications if compared to standard population. Obesity, smoking history and anemia are known risk factors that commonly impair body-contouring procedures outcomes [28]. In addition, according to our previous experience, BMI greater than $30 \mathrm{~kg} / \mathrm{m}^{2}$ appeared to be negative influence scarring process. Furthermore, concomitant bodycontouring procedures seem to increase post-operative complications [29]. Greater BMI loss has been reported as risk factor for the need of longer incisions and operative time [30].

The present study focused on brachioplasty as one of the main surgeries requested by post-bariatric female patients and more frequently burdened by complications related to wound healing and scar quality.

Our outcomes are consistent with those reported for other field of surgery and post-bariatric abdominoplasty as well, confirming the role of piNPWT to manage high risk surgical wounds [19, 21, 22, 31]. This study suggests that a user-friendly device applied under garments adequately 


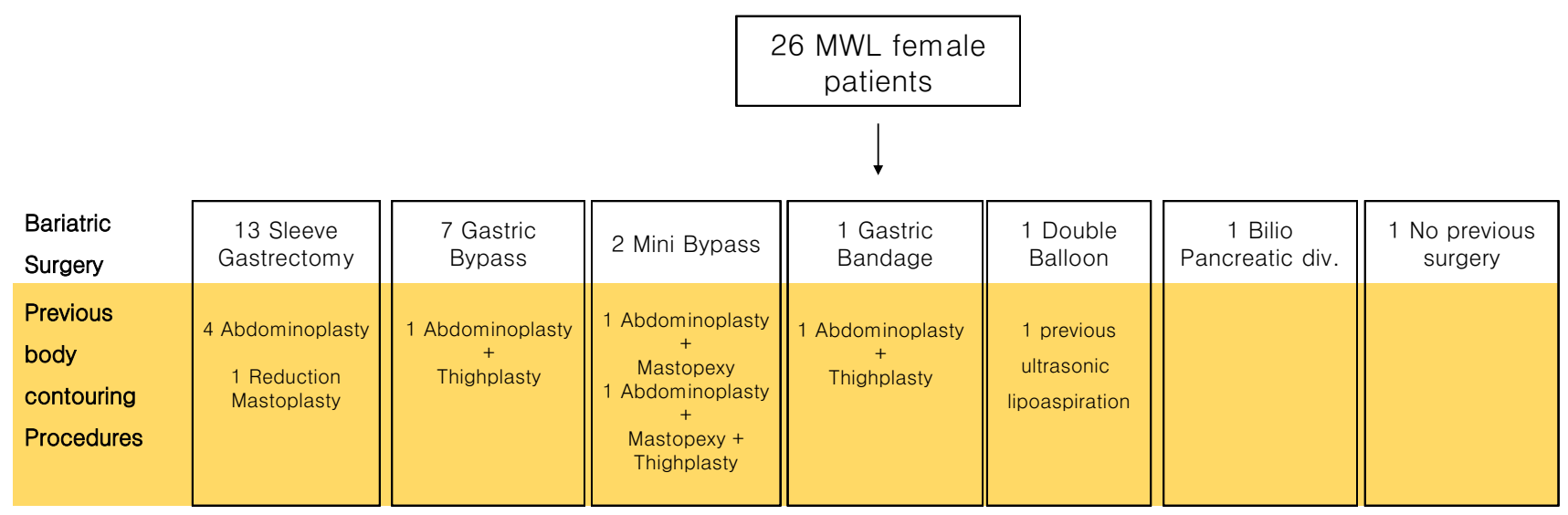

Fig. 5 Bariatric surgery and previous body-contouring procedures performed

Table 2 Post-operative outcomes of the two groups

\begin{tabular}{lll}
\hline & piNPWT & TDD \\
\hline Number of post-operative complication & 2 & 6 \\
Claviend-Dindo Grade I & 1 & 3 \\
Claviend-Dindo Grade II & $1 \mathrm{~s}$ & 2 \\
Claviend-Dindo Grade III & 0 & 1 \\
Number of SSI & None & None \\
Hypercromic scars 90 days after surgery & 7 & 0 \\
Post op. dressing change** & $2 \pm 0.77$ & $4.91 \pm 0.79$ \\
Time to dry (days)** & $9.36 \pm 2.15$ & $17.66 \pm 4.79$ \\
Hosp. Length (days)** & $3.07 \pm 114$ & $5.33 \pm 1.49$ \\
\hline
\end{tabular}

$* * p<0.05 \%$

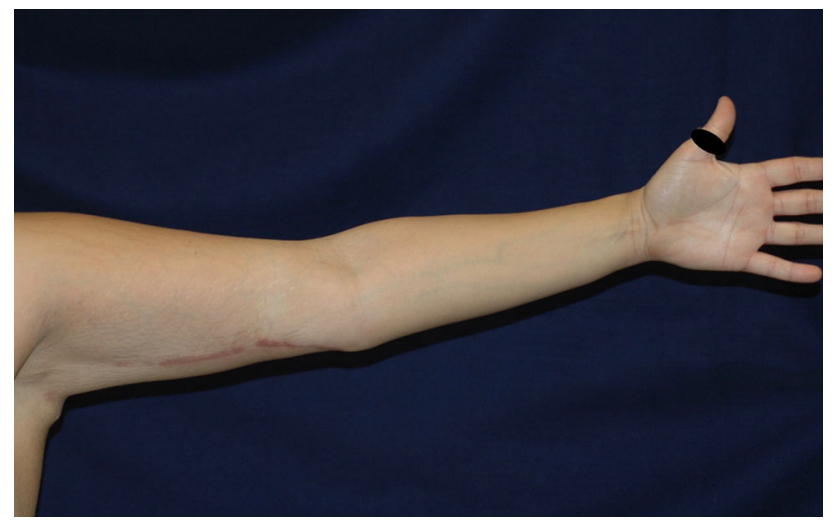

Fig. 6 Post-operative result 90 days after surgery in a patient of the piNPWT group who developed an hyperchromic scar

limits the number of complications without eliciting treatment-related cutaneous damage or causing patient discomfort responsible of premature dressing change. However, no beneficial effect was observed in preventing major complication Clavien-Dindo grade III [26]
The ability of the piNPWT to reduce the lateral tense of closed incision improved wound healing in our patients [32]. The role of the piNPWT in enhancing the reabsorption of tumescent solution after liposuction should be further investigated in MWL patients treated with the combination of lifting and liposuction.

The combination of body-contouring procedures is considered a risk factor for potential increasing of complication. Even if the combination of mastopexy and brachioplasty is a general favorable combination of procedures, the decision should be made evaluating patient's expectations, BMI loss, comorbidities and smoking status [33].

Among our groups, the higher number of patients undergoing a combination of mastopexy and brachioplasty in piNPWT sample ( 8 vs. 2 ) may have negatively influenced the post-operative course of the patients and the wound healing process. On the contrary, the portable incision NPWT may have lessen the risk. Although the scarring was from the functional point of view extremely satisfactory and comparable in both groups, according to our previous experience [29], the hyperchromic scar is an 
aesthetic aspect that should not be underestimated, especially in post-bariatric female patients.

VL Young et al. [34] indicates that patients are highly concerned about scarring following routine surgery. Their work also shows that there are disparities in patient-clinician communication regarding expectations following surgery. Hence, patients should be accurately counsel, given the transient effect on hyperchromic scars, which can negatively influence from a psychological point of view the post-operative period of MWL patients [26].

The reduced number of post-operative dressing changes and shorter extent of the hospital stay of piNPWT patients together with the reduction of local complications is known to impact favorably the cost management of this group of patients $[22,35]$.

Nonetheless, the presence of two dressing devices constantly attached to patients' arms could be annoying for patients. Even though, accordingly to the literature, nobody of piNPWT group complained about their dressing, patients should be accurately informed about the need to carry two devices hung to their arms for 7-12 days[36].

In addition, in the middle of the COVID-19 era, the reduction of the hospital stay, number of outpatient visits and complications allow limiting the exposure of patients to the risk of Sars-Cov2 infection [37, 38].

Our preliminary report, even if retrospectively, confirms the role of piNPWT in improving postoperative management of post-bariatric patient undergoing body-contouring procedures. Other comparative studies are needed to validate this approach in breast lifts and thigh lift. Further research has to be carried out before routine clinical adoption of this technique in particular to find the ideal group of patients that will benefit most (i.e., combination of body-contouring procedures or patient undergoing liposuction and lifting).

As people heal differently, a prospective comparison of the effect of the application piNPWT in one arm and traditional dressing in the other arm of a single patient could be an option to limit inter-patients variability.

Furthermore, a more comprehensive analysis of the costeffectiveness of the procedure should also be conducted.

\section{Conclusions}

In conclusion, we demonstrate that piNPWT is an effective medical tool, able to limit minor local complications rates in post-bariatric patients undergoing a brachioplasty.

The piNPWT represents a valid alternative to the traditional dry dressing. Given the transiently worrisome aesthetic result, informing the patient about the possible development of a hyperchromic scar is key. We suggest the use of this device in post-bariatric patients who undergo a brachioplasty, especially if there is the need to minimize the number of post-operative dressing changes or outpatients' visits and when brachioplasty is combined with liposuction and/or mastopexy.

Funding Open Access funding provided by Università degli Studi di Padova. No funding has been received in support of the paper

\section{Compliance with Ethical Standards}

Conflict of interest The authors have no conflict of interest regarding the submitted work

Ethical Approval The displayed study was carried out with respect of high ethical standards. All the studies have been approved, when required, by the appropriate ethics committee and have, therefore, been performed in accordance and in conformity to the World Medical Association Declaration of Helsinki (June 1964) and subsequent amendments.

Informed Consent All patients signed an informed consent for the procedures. For this type of study, formal consent is not required.

Open Access This article is licensed under a Creative Commons Attribution 4.0 International License, which permits use, sharing, adaptation, distribution and reproduction in any medium or format, as long as you give appropriate credit to the original author(s) and the source, provide a link to the Creative Commons licence, and indicate if changes were made. The images or other third party material in this article are included in the article's Creative Commons licence, unless indicated otherwise in a credit line to the material. If material is not included in the article's Creative Commons licence and your intended use is not permitted by statutory regulation or exceeds the permitted use, you will need to obtain permission directly from the copyright holder. To view a copy of this licence, visit http://creativecommons. org/licenses/by/4.0/.

\section{References}

1. Hruby A et al (2016) Determinants and consequences of obesity. Am J Public Health 106(9):1656-1662

2. Arterburn DE et al (2015) Association between bariatric surgery and long-term survival. JAMA 313(1):62-70

3. Froylich D et al (2016) Laparoscopic versus open ventral hernia repair in obese patients: a long-term follow-up. Surg Endosc 30(2):670-675

4. Araco A et al (2006) Body contouring after weight loss: the plastic-bariatric surgery symbiosis. Aesthet Plast Surg 30(3):374-376

5. Song AY et al (2006) Body image and quality of life in post massive weight loss body contouring patients. Obesity 14(9):1626-1636

6. Botero AG, Wenninger MG, Loaiza DF (2017) Complications after body contouring surgery in postbariatric patients. Ann Plast Surg 79(3):293-297

7. Gusenoff JA, Rubin JP (2008) Plastic surgery after weight loss: current concepts in massive weight loss surgery. Aesthet Surg J 28(4):452-455

8. Song AY et al (2005) A classification of contour deformities after bariatric weight loss: the Pittsburgh Rating Scale. Plast Reconstr Surg 116(5):1535-1544 
9. Sjöström L et al (2004) Lifestyle, diabetes, and cardiovascular risk factors 10 years after bariatric surgery. $\mathrm{N}$ Engl $\mathrm{J}$ Med 351(26):2683-2693

10. Macchi V et al (2014) Anatomical remodelling of the anterior abdominal wall arteries in obesity. Clin Hemorheol Microcirc 57(3):255-265

11. van der Beek ES et al (2013) Classification of contour deformities after massive weight loss: the applicability of the Pittsburgh Rating Scale in The Netherlands. J Plast Reconstr Aesthet Surg 66(8):1039-1044

12. Rubin JP, Nguyen V, Schwentker A (2004) Perioperative management of the post-gastric-bypass patient presenting for body contour surgery. Clin Plast Surg 31(4):601-610

13. El Khatib HA (2007) Classification of brachial ptosis: strategy for treatment. Plast Reconstr Surg 119(4):1337-1342

14. Surgeons, ASoP, National Plastic Surgery Statistics: cosmetic and reconstructive procedure trends. News and Resources - 2009 Statistics, 2000/2008/2009

15. Vindigni $\mathrm{V}$ et al (2019) The posterior arm flap for reshaping the postbariatric breast. Plast Reconstr Surg Glob Op. https://doi.org/ 10.1097/GOX.0000000000002434

16. Joseph Michaels V, Coon D, Rubin JP (2011) Complications in postbariatric body contouring: postoperative management and treatment. Plast Reconstr Surg 127(4):1693-1700

17. Jørgensen MG et al (2018) Prevention of seroma following inguinal lymph node dissection with prophylactic, incisional, negative-pressure wound therapy (SEROMA trial): study protocol for a randomized controlled trial. Trials 19(1):1-8

18. Kim DY et al (2016) Does the use of incisional negative-pressure wound therapy prevent mastectomy flap necrosis in immediate expander-based breast reconstruction? Plast Reconstr Surg 138(3):558-566

19. Mangelsdorff $G$ et al (2019) Reduced anterolateral thigh flap donor-site morbidity using incisional negative pressure therapy. J Reconstr Microsurg 35(03):229-234

20. Hyldig $N$ (2016) Incisional negative pressure wound therapy the clinical effect on post-caesarean wound complications in obese women. Syddansk Universitet, Denmark

21. Echebiri NC et al (2015) Prophylactic use of negative pressure wound therapy after cesarean delivery. Obstet Gynecol 125(2):299-307

22. Abatangelo S, Saporiti E, Giatsidis G (2018) Closed incision negative-pressure therapy (ciNPT) reduces minor local complications in post-bariatric abdominoplasty body contouring: a retrospective case-control series. Obes Surg 28(7):2096-2104

23. Hurwitz DJ, Neavin T (2008) L brachioplasty correction of excess tissue of the upper arm, axilla, and lateral chest. Clin Plast Surg 35(1):131-140

24. Reed LS (2014) Brachioplasty with limited scar. Clin Plast Surg 41(4):753-763
25. Simone P et al (2018) Postbariatric brachioplasty with posteromedial scar: physical model, technical refinements, and clinical outcomes. Plast Reconstr Surg 141(2):344-353

26. Clavien PA et al (2009) The Clavien-dindo classification of surgical complications: five-year experience. Ann Surg 250(2):187-196

27. Draaijers LJ et al (2004) The patient and observer scar assessment scale: a reliable and feasible tool for scar evaluation. Plast Reconstr Surg 113(7):1960-1965

28. Coon D et al (2013) Plastic surgery and smoking: a prospective analysis of incidence, compliance, and complications. Plast Reconstr Surg 131(2):385-391

29. Marchica P et al (2020) Retrospective analysis of the predictive factors associated with good surgical outcome in brachioplasty in massive weight loss patients. J Plast Surg Hand Surg. https://doi. org/10.1080/2000656X.2020.1788043

30. Coon D et al (2009) Body mass and surgical complications in the postbariatric reconstructives patient: analysis of 511 cases. Ann Surg 249(3):397-401

31. Dragu A et al (2011) Wide topical negative pressure wound dressing treatment for patients undergoing abdominal dermolipectomy following massive weight loss. Obes Surg 21(11):1781-1786

32. Loveluck $\mathrm{J}$ et al (2016) Biomechanical modeling of the forces applied to closed incisions during single-use negative pressure wound therapy. Eplasty 16:e20

33. Almutairi K, Gusenoff JA, Rubin JP (2016) Body contouring. Plast Reconstr Surg 137(3):586e-602e

34. Young VL, Hutchison J (2009) Insights into patient and clinician concerns about scar appearance: semiquantitative structured surveys. Plast Reconstr Surg 124(1):256-265

35. Chopra $\mathrm{K}$ et al (2016) The economic impact of closed-incision negative-pressure therapy in high-risk abdominal incisions: a cost-utility analysis. Plast Reconstr Surg 137(4):1284-1289

36. Scalise A et al (2016) Improving wound healing and preventing surgical site complications of closed surgical incisions: a possible role of incisional negative pressure wound therapy. A systematic review of the literature. Int Wound J 13(6):1260-1281

37. Kaye $\mathrm{K}$ et al (2020) Elective, Non-urgent procedures and aesthetic surgery in the wake of SARS-COVID-19: Considerations regarding safety feasibility and impact on clinical management. Aesthet Plast Surg. https://doi.org/10.1007/s00266-020-01752-9

38. The reorganization of the strategies of a surgical department and the nosocomial diffusion of Covid-19

Publisher's Note Springer Nature remains neutral with regard to jurisdictional claims in published maps and institutional affiliations. 